\title{
Podofilox suppresses gastric cancer cell proliferation by regulating cell cycle arrest and the c-Myc/ATG10 axis
}

\author{
JUAN AN $^{1,2}$, YAN LIU ${ }^{1}$, SHENGLAN DUO $^{3}$, XIAOMING MA $^{4}$, LING AN $^{5}$, YUNFEI YAN $^{1}$, \\ DONGDE JI ${ }^{5}$, YUPENG YAN ${ }^{1}$, QILIANG CHENG $^{1}$ and ZHANHAI SU ${ }^{1,2}$ \\ ${ }^{1}$ Department of Basic Medical Sciences, Qinghai University Medical College; \\ ${ }^{2}$ State Key Laboratory of Plateau Ecology and Agriculture, Qinghai University, Xining, Qinghai 810016; \\ ${ }^{3}$ Department of Doppler Ultrasound, Qinghai Red Cross Hospital, Xining, Qinghai 810099; \\ ${ }^{4}$ Department of Gastrointestinal Tumor Surgery, The Affiliated Hospital of Qinghai University, Xining, \\ Qinghai 810012; ${ }^{5}$ Department of Internal Medicine, Qinghai People's Hospital, Xining, Qinghai 810007, P.R. China
}

Received October 17, 2020; Accepted June 21, 2021

DOI: $10.3892 /$ etm.2021.10637

\begin{abstract}
Gastric cancer (GC) is a malignancy for which effective therapeutic drugs are limited. Podofilox exhibits antitumor effects in various types of cancer; however, whether it may inhibit GC growth remains unknown. The aim of the present study was to investigate the role of podofilox in GC. Cell Counting Kit-8, colony formation and cell cycle assays were used to detect the role of podofilox on cellular proliferation and the cell cycle, respectively. A microarray was used to detect the transcriptional changes induced by podofilox in GC cells. The results of the present study demonstrated that podofilox inhibited GC cell proliferation and colony formation. The half maximal inhibitory concentration of podofilox in AGS and HGC-27 cells was 2.327 and $1.981 \mathrm{nM}$, respectively. In addition, treatment with podofilox induced $\mathrm{G}_{0} / \mathrm{G}_{1}$ cell cycle arrest. Molecular analysis based on microarray data demonstrated that podofilox altered the expression levels of genes involved in the cell cycle, c-Myc and p53 signaling. Autophagy-related 10 (ATG10), which was highly expressed in GC tissues, was also downregulated by podofilox, as demonstrated by the results of the microarray analysis and immunoblotting. To determine the involvement of ATG10 in GC, ATG10 was knocked down in GC cells by small interfering RNA, which suppressed the proliferation and colony formation of GC cells compared with those observed in the control-transfected cells. Taken together, the results of the present study suggested that podofilox may inhibit GC cell proliferation
\end{abstract}

Correspondence to: Professor Juan An or Professor Zhanhai Su, Department of Basic Medical Sciences, Qinghai University Medical College, 251 Ningzhang Road, Xining, Qinghai 810016, P.R. China E-mail: anjuan@qhu.edu.cn

E-mail: suzhanhai@qhu.edu.cn

Key words: podofilox, gastric cancer, cell cycle, apoptosis, autophagy by preventing the cell cycle progression and regulating the c-Myc/ATG10 signaling pathway.

\section{Introduction}

Gastric cancer (GC) is one of the most common cancer types, which originates from the gastric mucosa, neuroendocrine, lymphoid or connective tissue of the gastric wall (1). Helicobacter pylori infection $(1,2)$, cigarette smoking (3), alcohol (4) and genetic syndromes (5) are common risk factors for GC. Surgical resection is the optimal therapeutic option for GC. Increasing evidence has demonstrated that genetic variants, such as those in p53, adenomatous polyposis coli and human epidermal growth factor receptor-2 (HER-2), contribute to the development of GC $(6,7)$. Targeted therapies against HER-2 have been applied for the treatment of GC patients with HER-2 amplification (8). However, there is still a demand to identify novel biomarkers and triggers of GC progression.

Podofilox, which is isolated from the North American plant Podophyllum peltatum L., belongs to the lignan family, and one of its derivatives is a potential antitumor factor (9). This natural product has also been isolated from Podophyllum emodi (9). According to previous studies, podofilox derivatives exhibit inhibitory effects on the proliferation and growth of various types of cancer. For example, XWL-1-48, a novel podofilox derivative, induces apoptosis and suppresses cell proliferation in breast cancer (10). Another derivative, $4 \beta-\mathrm{NH}$-(benzo heterocycles)-4-desoxy-podofilox, exhibits anticancer effects by improving tubulin binding affinity in MCF-7, A549 and HeLa cells (11).

Currently, the effects of podofilox and its potential underlying molecular mechanism of action in GC remain unclear. In this context, the present study aimed to investigate whether podofilox may exert an anticancer effect in GC, and the potential molecular mechanisms therein.

\section{Materials and methods}

The Cancer Genome Atlas (TCGA) data analysis. For the analysis of public data, 375 GC tissue and non-cancer 
tissues were analyzed using a dataset from (TCGA) database (http://cancergenome.nih.gov).

Cell lines and culture. The human GC cell lines AGS and HGC-27 were purchased from the American Type Culture Collection and maintained according to the supplier's instructions. Cells were maintained in DMEM supplemented with $10 \%$ fetal bovine serum (Gibco; Thermo Fisher Scientific, Inc.) and $1 \%$ penicillin/streptomycin at $37^{\circ} \mathrm{C}$ with $5 \% \mathrm{CO}_{2}$.

IC50 measurement. The $\mathrm{IC}_{50}$ of podofilox was determined using the Cell Counting Kit-8 (CCK-8; Shanghai Yeasen Biotechnology Co., Ltd.) assay. GC cells were seeded into 96-well plates in triplicate at a density of 2,000 cells per well and treated with various concentrations $(0,0.3125,0.625$, $1.25,2.5,5,10,20,40$ and $80 \mathrm{nM}$; dissolved in DMSO) of podofilox (cat. no. S18-28-5; MedChemExpress). The plates were maintained at $37^{\circ} \mathrm{C}$ with $5 \% \mathrm{CO}_{2}$. The CCK-8 assay was subsequently performed to assess cell viability after $48 \mathrm{~h}$. The culture medium was removed, and $100 \mu$ l culture medium was added to each well with $10 \mu \mathrm{l}$ Cell CCK-8 reagent (Beyotime Institute of Biotechnology). After incubation for $3 \mathrm{~h}$ at room temperature, cell viability was analyzed by measuring the $\mathrm{OD}$ at $450 \mathrm{~nm}$, using a microplate reader (Thermo Fisher Scientific, Inc.).

ATG10 interference. Small interfering (si)RNAs against ATG10 (siATG10)-1 (5'-GAGUUCAUGAGUGCUAUAAGA-3') and siATG10-2 (5'-GCAACAGGAACAUCCAAUACU-3') and a control siRNA (siCtrl; 5'-UUCUCCGAACGUGUCACG U-3') were synthesized by Huzhou Hippo Biotechnology Co., Ltd. A total of $3 \times 10^{5}$ cells were seeded in 6-well plates and transfected with siRNAs $\left(50 \mathrm{nM} /\right.$ well) using Lipofectamine ${ }^{\circledR}$ RNAiMAX reagent ( $4 \mu \mathrm{l} /$ well; Thermo Fisher Scientific, Inc.) for $48 \mathrm{~h}$ at $37^{\circ} \mathrm{C}$, according to the manufacturer's protocol. At $48 \mathrm{~h}$ post-transfection, knockdown efficacy was determined by western blotting and the cells were subjected to cell function experiments.

Cell viability assay. A total of $2 \times 10^{3} /$ well AGS and HGC-27 cells were seeded into 96-well plates containing $100 \mu \mathrm{l}$ DMEM (10\% FBS) and treated with $3.4 \mathrm{nM}$ podofilox at $37^{\circ} \mathrm{C}$ for $1,2,3,4$ and 5 days. CCK-8 reagent $(10 \%)$ was subsequently added to each well and cellular proliferation was analyzed at a wavelength of $450 \mathrm{~nm}$ after incubation for $3 \mathrm{~h}$ at $37^{\circ} \mathrm{C}$.

Colony formation assay. AGS and HGC-27 cells were seeded at a density of 1,000 cells/well and incubated at $37^{\circ} \mathrm{C}\left(5 \% \mathrm{CO}_{2}\right)$ with vehicle or $3.4 \mathrm{nM}$ podofilox. The culture medium, vehicle and podofilox were changed every 2 days. After 7 days, the colonies were washed with PBS, fixed with $100 \%$ methanol at room temperature for $15 \mathrm{~min}$, and then stained with $0.2 \%$ crystal violet solution at room temperature for $30 \mathrm{~min}$. Images were captured using a camera (Nikon Corporation), and cell colonies $>50$ cells were counted manually.

Western blotting. AGS and HGC-27 cells treated with or without podofilox were lysed using a lysis buffer (Beyotime Institute of Biotechnology). The concentration of total protein was detected using a BCA Protein Assay Kit. Proteins (30 $\mu \mathrm{g})$ were separated by SDS-PAGE (12\%), transferred on PVDF membranes (MilliporeSigma) and blocked with non-fat milk at room temperature for $1 \mathrm{~h}$. The membranes were incubated with primary antibodies against hexokinase-2 (HK2; cat. no. 2867; Cell Signaling Technology, Inc.), pyruvate kinase M1/2 (PKM2; cat. no. 4053; Cell Signaling Technology, Inc.), ATG10 (cat. no. ab124711; Abcam), p53 (cat. no. sc-126; Santa Cruz Biotechnology, Inc.), c-Myc (cat. no. sc-40; Santa Cruz Biotechnology, Inc.) and GAPDH (cat. no. sc-47724; Santa Cruz Biotechnology, Inc.) at $4^{\circ} \mathrm{C}$ overnight. Following primary antibody incubation, the membranes were washed with TBST $(0.1 \%$ Tween $)$ three times at room temperature, and incubated with secondary antibodies (Santa Cruz Biotechnology, Inc. both 1:10,000; normal mouse IgG, cat. no. sc-2748; and normal rat IgG, cat. no. sc-2750) at room temperature for $3 \mathrm{~h}$. Protein bands were visualized using SuperSignal $^{\mathrm{TM}}$ West Pico PLUS Chemiluminescent Substrate (Thermo Fisher Scientific, Inc.). The western blot bands were quantified using ImageJ software (v1.8.0).

Cell cycle analysis. AGS and HGC-27 cells $\left(1 \times 10^{5}\right)$ were seeded and incubated with vehicle or podofilox $(3.4 \mathrm{nM})$ for $48 \mathrm{~h}$. The cells were collected and incubated with $70 \%$ iced ethanol overnight. Cell cycle distribution was assessed using the propidium iodide (Shanghai Yeasen Biotechnology Co., Ltd.) staining kit, according to the manufacturers' protocols, and analyzed via flow cytometry.

Microarray analysis. Total RNA was extracted from AGS cells treated with vehicle or podofilox $(3.4 \mathrm{nM})$ using TRIzol ${ }^{\circledR}$ reagent (Invitrogen; Thermo Fisher Scientific, Inc.) according to the manufacturer's protocol. The quality and quantity of the RNA were determined using NanoDrop 2000. PrimeView Human Gene Expression Array (Affymetrix, cat. no. 902487) was used to profile gene expression. The following criteria were used to determine statistical significance: $\mathrm{P}<0.05$ and fold-change $>1.3$. The Kyoto Encyclopedia of Genes and Genomes (KEGG) and HALLMARK databases were used to perform pathway enrichment analyses (12).

Statistical analysis. Statistical analysis was performed using GraphPad prism 8.0 software (GraphPad Software, Inc.). All experiments were performed in triplicate and data are presented as the mean \pm standard error of the mean. Student's t-test was used to compare differences between two groups, whereas one way ANOVA followed by Tukey's post hoc test were used to compare differences among multiple groups. $\mathrm{P}<0.05$ was considered to indicate a statistically significant difference.

\section{Results}

Podofilox effectively inhibits cell proliferation. To study the effects of podofilox in $\mathrm{GC}$ cells, the $\mathrm{IC}_{50}$ of podofilox was determined. AGS and HGC-27 cells were treated with various concentrations of podofilox ranging between 0 and $80 \mathrm{nM}$, and the cell viability was determined at $48 \mathrm{~h}$ post-treatment. The results demonstrated that $50 \%$ of AGS and HGC-27 cells died following incubation with 
A

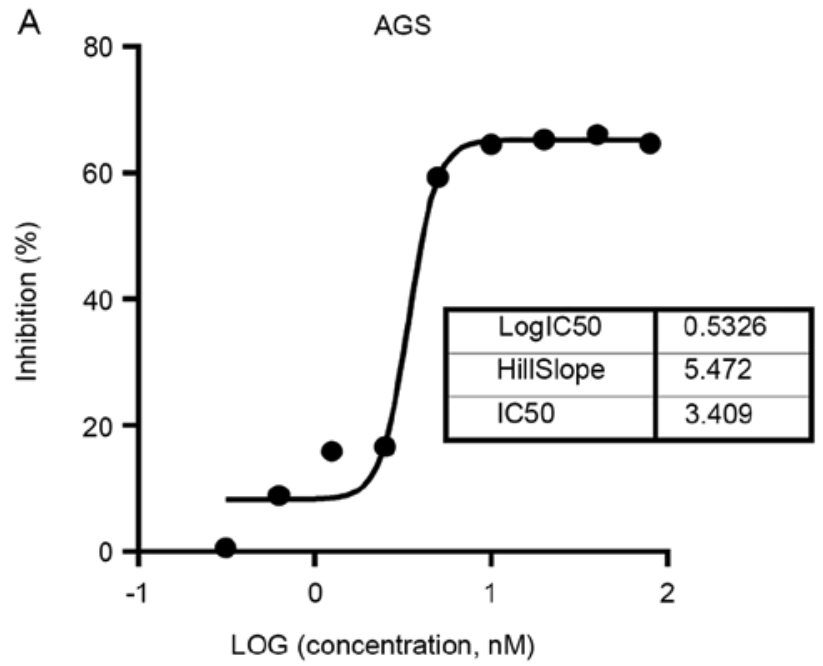

C

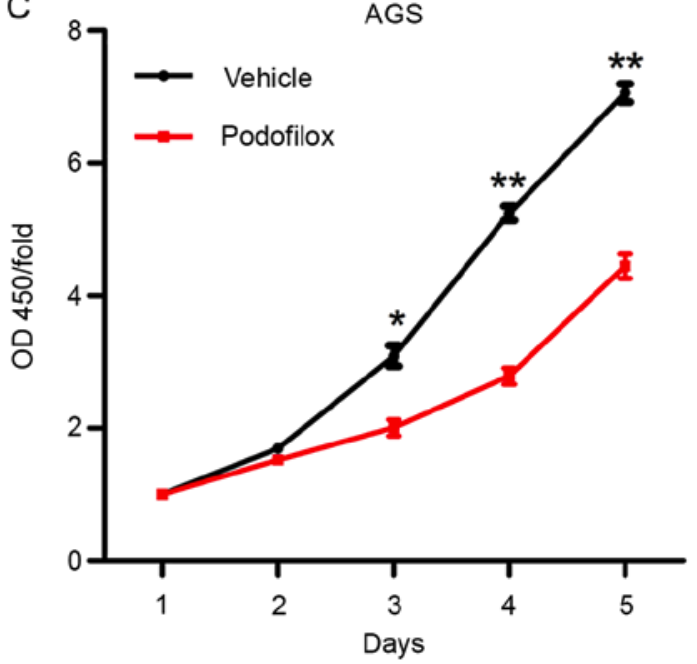

B

B 100

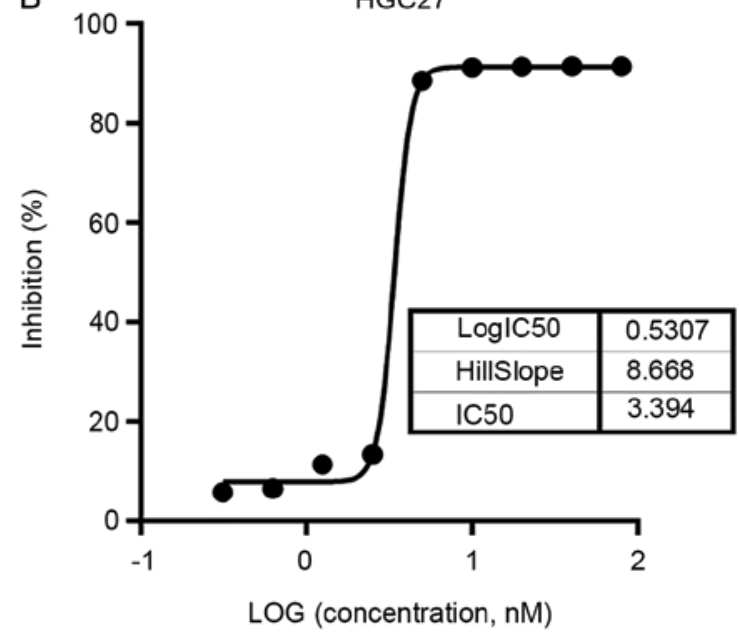

HGC27

D

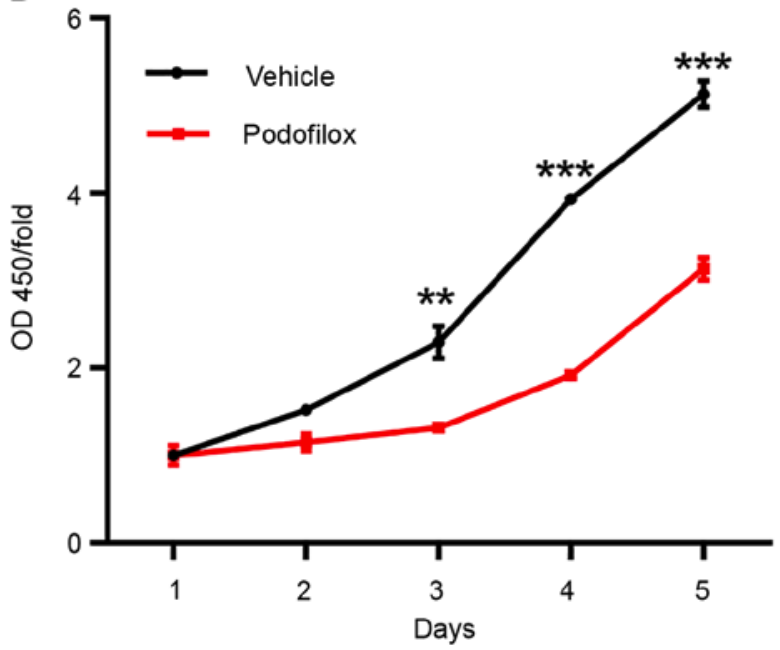

Figure 1. Podofilox inhibits the proliferation of HGC-27 and AGS cells. (A and B) Cell viability was assessed in (A) AGS and (B) HGC-27 cells following incubation with podofilox $(0-80 \mathrm{nM})$ for $48 \mathrm{~h}$. (C and D) Cell proliferation curve was analyzed following incubation with vehicle or $3.4 \mathrm{nM}$ podofilox at the indicated time points. ${ }^{*} \mathrm{P}<0.05,{ }^{* * *} \mathrm{P}<0.01$ and $^{* * *} \mathrm{P}<0.001$ vs. vehicle.

3.409 and $3.394 \mathrm{nM}$ podofilox, respectively (Fig. 1A and B). Subsequently, the present study investigated whether podofilox exhibited a time-dependent inhibitory effect on cell proliferation. GC cells were incubated with vehicle or $3.4 \mathrm{nM}$ podofilox. Cell viability was analyzed between days 1 and 5. The results of the CCK-8 assay demonstrated that $3.4 \mathrm{nM}$ podofilox significantly suppressed cell proliferation compared with that observed in the vehicle-treated cells (Fig. 1C and D). Consistent with these results, cell colony formation was inhibited following treatment with podofilox (Fig. 2A and B). Taken together, these results suggested that podofilox effectively suppressed the proliferation and GC cells for 5 days.

Podofilox inhibits cell cycle progression. Next, the cell cycle was analyzed following treatment with vehicle or podofilox by PI staining and flow cytometric analysis. As presented in Fig. 3A and B, treatment with podofilox significantly increased the numbers of AGS and HGC-27 cells in the non-proliferative $\mathrm{G}_{0} / \mathrm{G}_{1}$ phase. These results suggested that podofilox promoted cell cycle arrest.
Molecular changes following treatment with podofilox. To determine the molecular mechanisms underlying the inhibitory effects of podofilox in GC, the cells were treated with vehicle and podofilox for $48 \mathrm{~h}$, and total RNA was extracted for microarray analysis. A total of 566 upregulated and 713 downregulated genes were identified following treatment with podofilox (Fig. 4A and Table S1). The expression levels of ATG10, an autophagy-related gene, were suppressed by podofilox treatment compared with those detected in the vehicle control group. Based on pathway enrichment analysis, 'KEGG_ cell_cycle' and 'Hallmark_MYC_Targets_v1' pathways were also suppressed by podofilox, while 'Hallmark_p53_Pathway' was activated by podofilox (Fig. 4B).

To validate the microarray results, western blot analysis was performed, and the results demonstrated that compared with the protein levels in the vehicle control group, podofilox downregulated the protein expression levels of HK2, PKM2, c-Myc and ATG10, and upregulated those of p53 (Fig. 5). Taken together, these results suggested that podofilox may suppress the cell cycle and the c-Myc/ATG10 axis, and activate $\mathrm{p} 53$. 
A

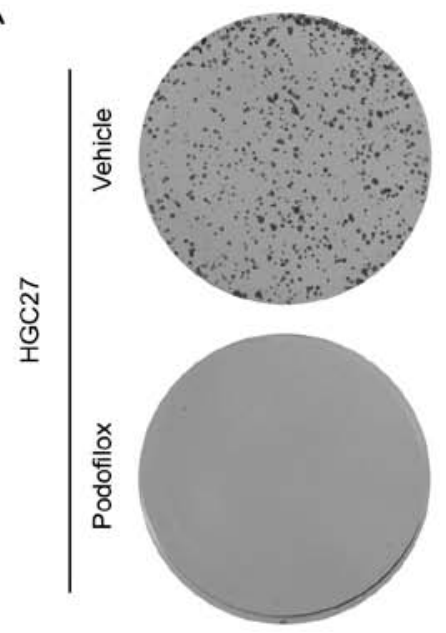

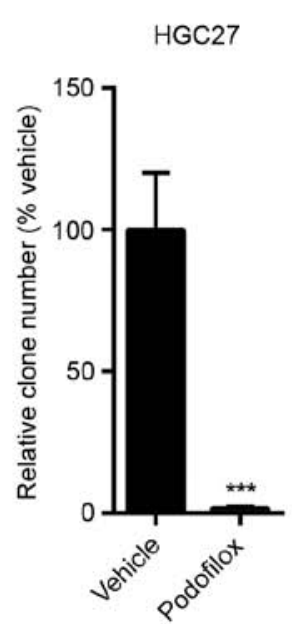

B

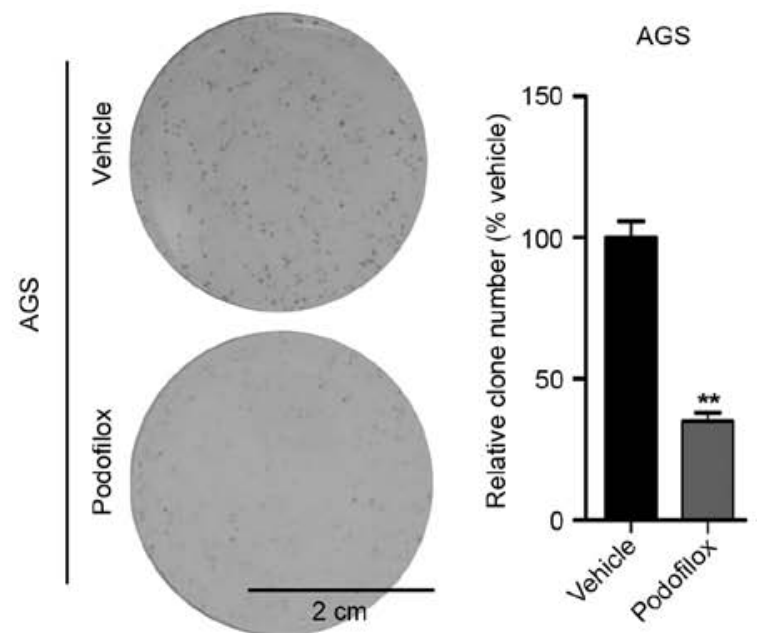

Figure 2. Podofilox inhibits cell colony formation. (A) AGS and (B) HGC-27 cells were incubated with vehicle or $3.4 \mathrm{nM}$ podofilox for 7 days, and cell colony formation was assessed. ${ }^{* *} \mathrm{P}<0.01$ and ${ }^{* * *} \mathrm{P}<0.001$ vs. vehicle.

A

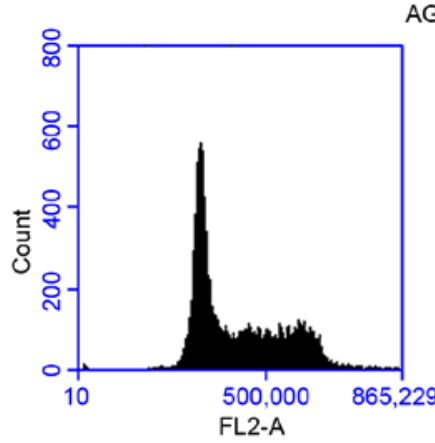

B

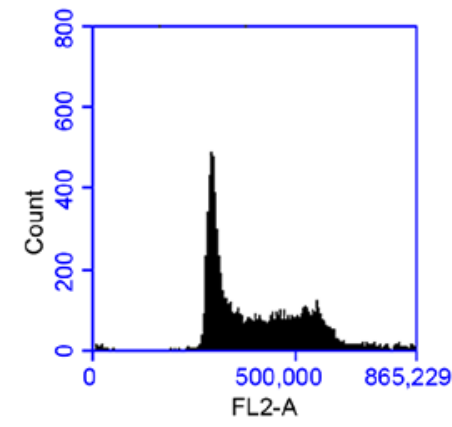

AGS

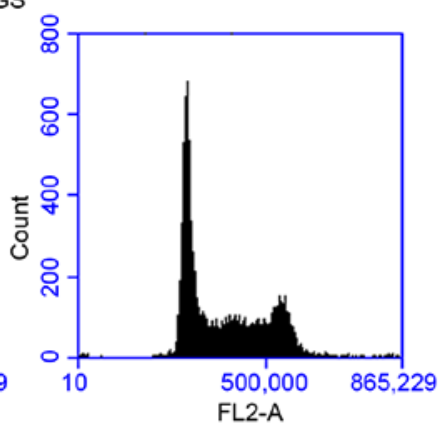

HGC-27

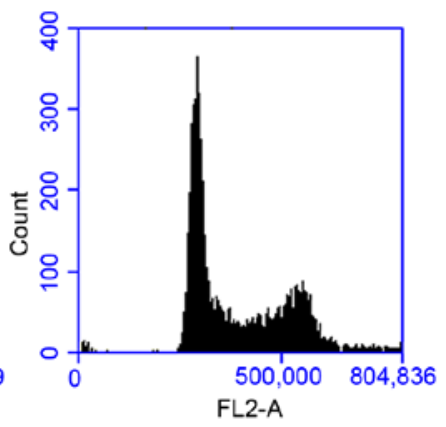

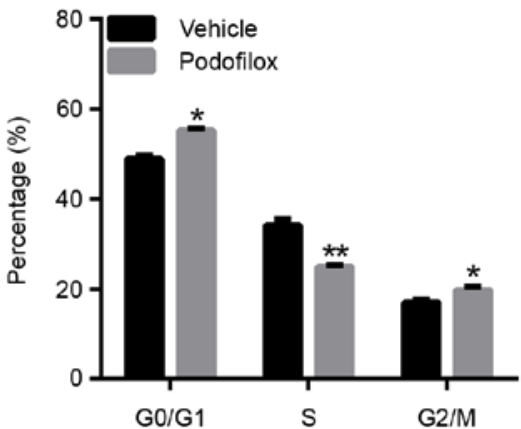

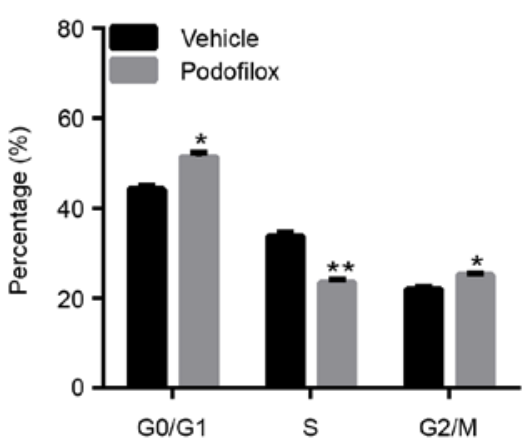

Figure 3. Podofilox inhibits cell cycle progression of HGC-27 and AGS cells. (A and B) The cell cycle was analyzed in (A) AGS and (B) HGC-27 cells following incubation with vehicle or $3.4 \mathrm{nM}$ podofilox for $48 \mathrm{~h}$. Left, representative images; right, quantification analysis. ${ }^{*} \mathrm{P}<0.05$ and ${ }^{* *} \mathrm{P}<0.01$ vs. vehicle.

ATG10 knockdown inhibits cell proliferation. The role of ATG10 in cell proliferation was subsequently investigated. The Cancer Genome Atlas (TCGA) database revealed that ATG10 was upregulated in GC samples compared with adjacent non-cancerous tissue (Fig. 6A). To determine the function of ATG10 in GC, ATG10 was silenced in GC cells, and proliferation was assessed by CCK-8 assay. Western blot analysis was performed to assess the transfection efficiency (Fig. 6B). The results demonstrated that ATG10 knockdown decreased the proliferation of HGC-27 cells compared with those in cells transfected with siCtrl (Fig. 6C). In AGS cells, ATG10 interference by siATG10-1 exhibited notable inhibition; however, siATG10-2 had a marginal inhibitory effect on the proliferation of AGS cells compared with that in the control group (Fig. 6D). The results of the colony formation assay demonstrated that both siRNAs targeting ATG10 significantly suppressed colony formation in GC cells (Fig. 6E and F). Thus, ATG10 may act as an oncogene in $\mathrm{GC}$.

\section{Discussion}

Advanced GC is an incurable malignancy as the current therapeutic strategies, including surgery, targeted therapies and immunotherapy, are ineffective against this disease (13). Despite recent advances, the prognosis of patients with GC 
A

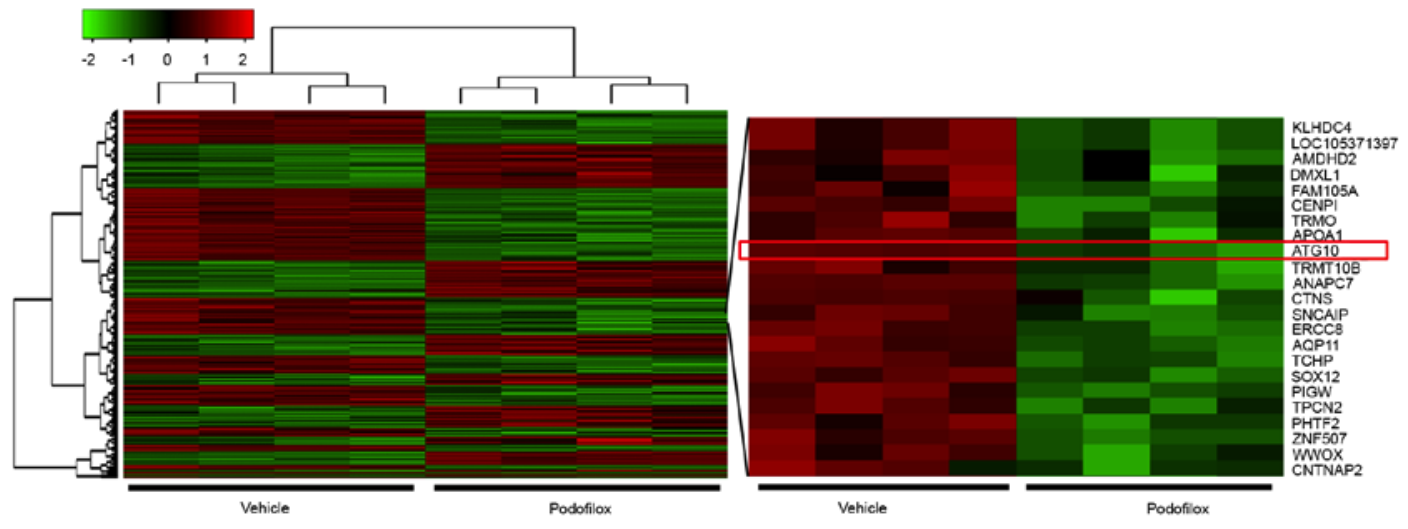

B
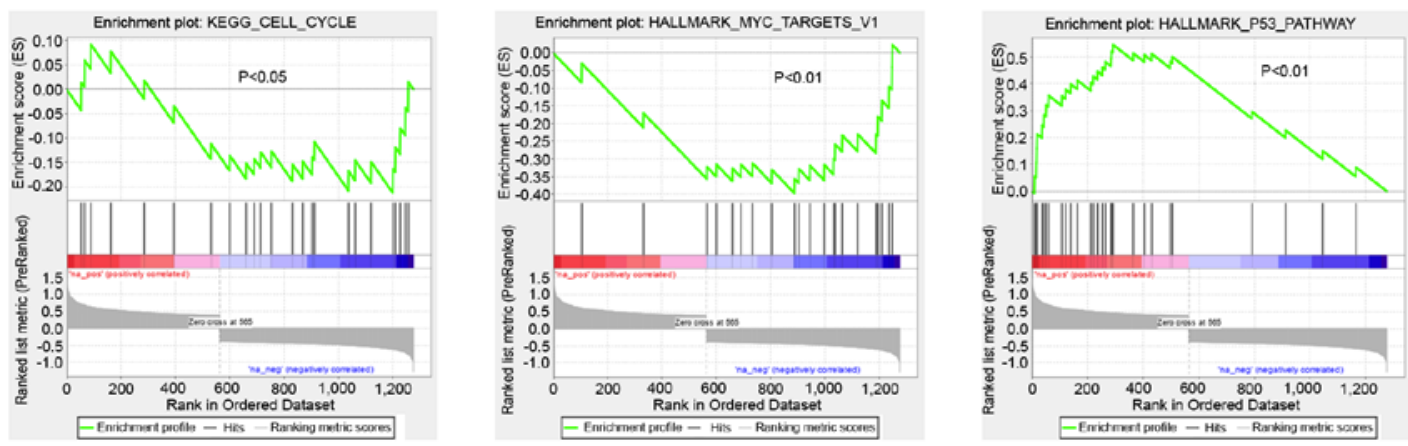

Figure 4. Microarray analysis of dysregulated genes following treatment with podofilox. (A) Heatmap of dysregulated genes in AGS cells treated with vehicle or podofilox. A total of 566 genes were upregulated, and 713 genes were downregulated. Green, downregulated genes; red, upregulated genes. (B) KEGG and HALLMARK pathway enrichment analyses of cell cycle, c-Myc and p53 signaling pathways. KEGG, Kyoto Encyclopedia of Genes and Genomes.
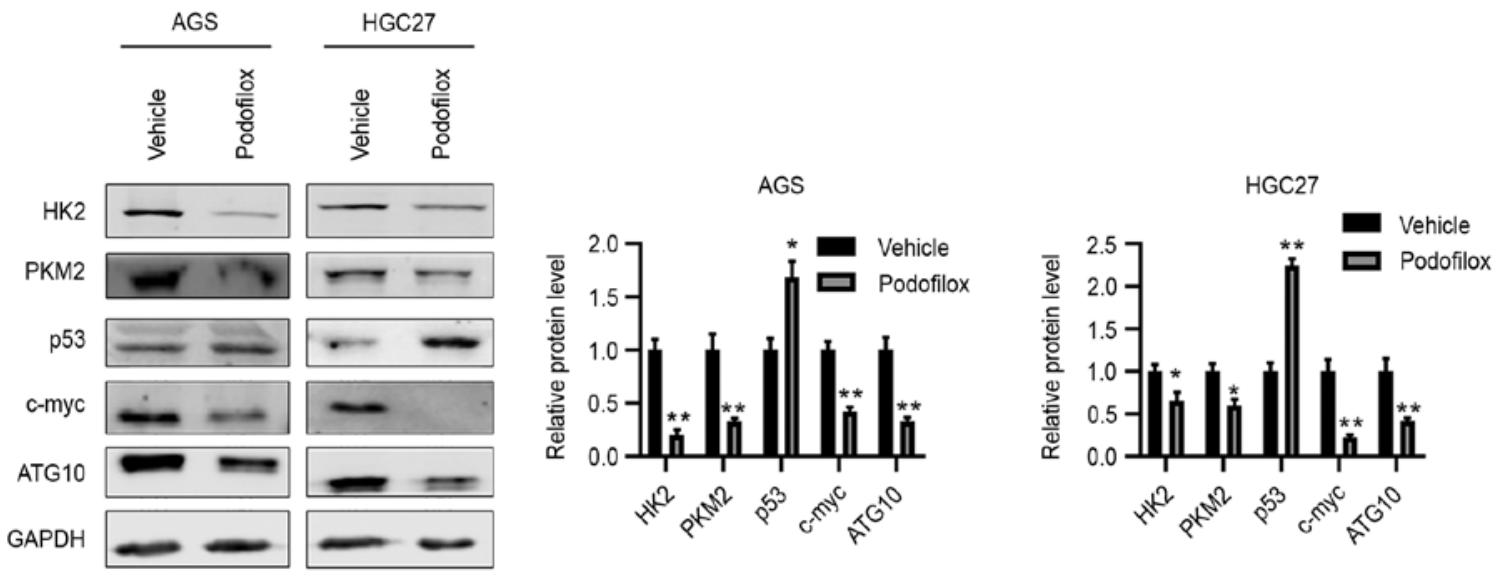

Figure 5. Western blot analysis of dysregulated genes following treatment with podofilox. Immunoblots of HK2, PKM2, p53, c-Myc, ATG10 and GAPDH following incubation with vehicle or $3.4 \mathrm{nM}$ podofilox for $48 \mathrm{~h}$ are presented on the left, and the quantitative analysis is presented on the right. " $\mathrm{P}<0.05$ and ${ }^{* *} \mathrm{P}<0.01$ vs. vehicle. HK2, hexokinase 2; PKM2, pyruvate kinase M1/2; ATG10, autophagy-related 10.

remains poor $(768,793$ new deaths in 2020), and effective treatment is limited (14). The results of the present study demonstrated that podofilox significantly suppressed the proliferative ability of GC cells. In addition, cell cycle arrest was induced by podofilox at the $\mathrm{G}_{0} / \mathrm{G}_{1}$ phase. Taken together, these results suggested that podofilox may be used as a therapeutic drug for the treatment of GC.

Podofilox, also termed podophyllotoxin, exhibits an antiviral function against herpes viruses, warts and influenza, as well as antitumor effects $(15,16)$. According to previous studies, its derivatives are widely used in different types of cancer. For example, dendrimer-conjugated podophyllotoxin suppresses the development of chemical-induced hepatocellular carcinoma (HCC) in mice (17). In another study, 14 biotinylated podophyllotoxin derivatives were designed and synthesized, which exhibited potent cytotoxic effects against a number of cancer cell lines, such as H1299 and H1975 (18). Furthermore, the $4 \beta$-acetamidobenzofuranone-podophyll otoxin hybrids exhibit anticancer effects in various types of cancer cells, such as breast, lung and prostate cancer (19). The results of the present study demonstrated that podofilox exhibited anticancer effects against GC cells. The $\mathrm{IC}_{50}$ of podofilox 
A

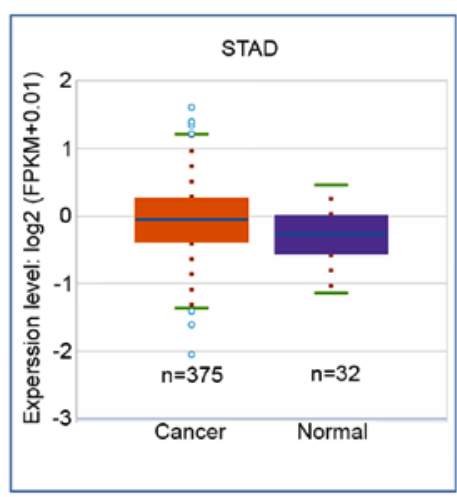

C

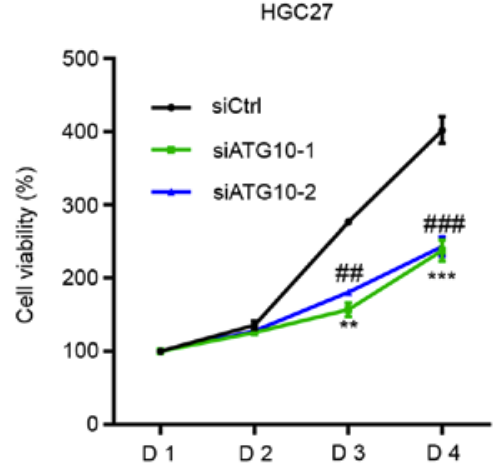

B

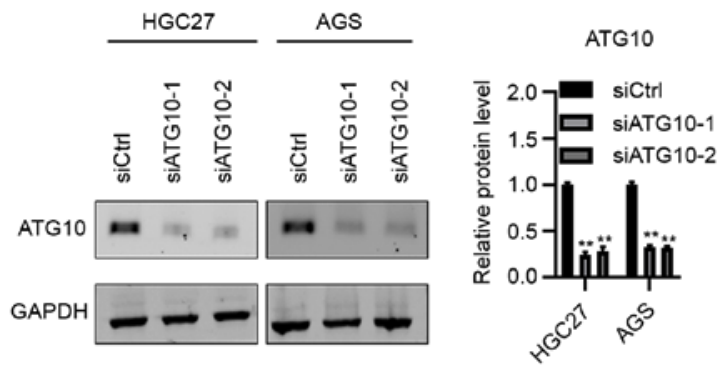

D

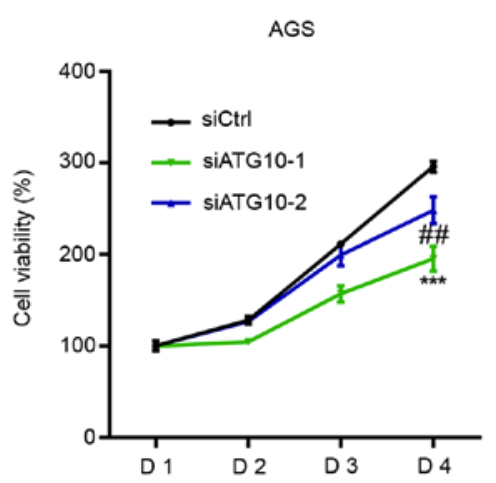

$E$

$\mathrm{F}$
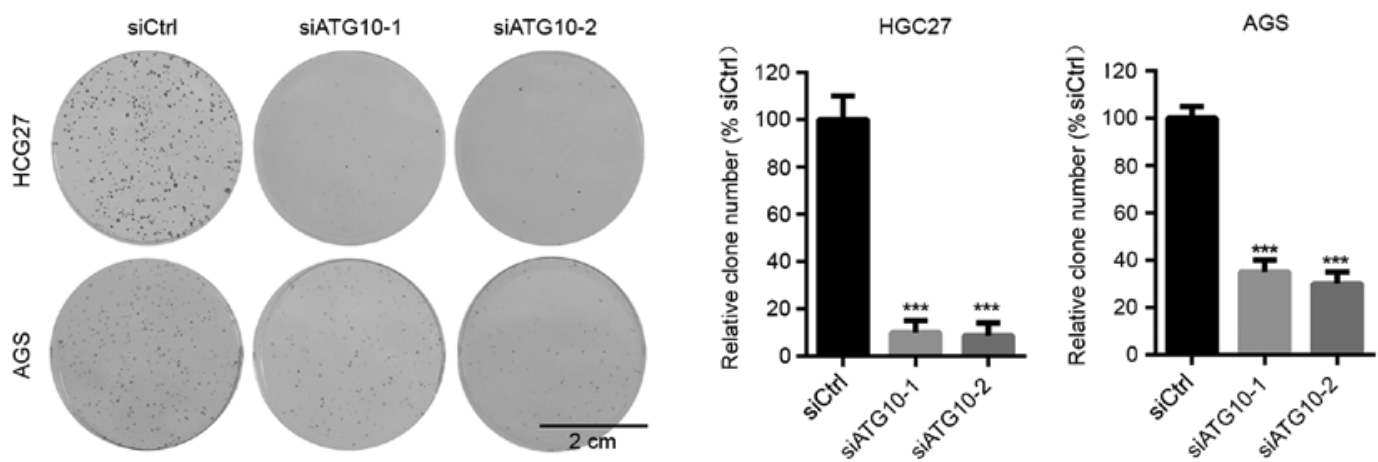

Figure 6. Downregulation of ATG10 inhibits cell proliferation and colony formation. (A) The expression levels of ATG10 in gastric cancer and normal tissues were analyzed using The Cancer Genome Atlas database. (B) Immunoblotting results of ATG10 and GAPDH in siCtrl-, siATG10-1- and siATG10-2-transfected HGC-27 and AGS cells (left) and the quantitative analysis (right). (C and D) Cell proliferation curves were analyzed in siCtrl-, siATG10-1- and siATG10-2-transfected cells. (E and F) Colony formation was assessed in siCtrl-, siATG10-1- and siATG10-2-transfected HGC-27 and AGS cells. (E) Images of colonies and (F) quantified data. ${ }^{* *} \mathrm{P}<0.05$ and ${ }^{* * *} \mathrm{P}<0.001$ siATG10-1 vs. siCtrl; ${ }^{\# \#} \mathrm{P}<0.01$ and ${ }^{\# \# \#} \mathrm{P}<0.001$ siATG10-2 vs. siCtrl. si, small interfering RNA; Ctrl, negative control; ATG10, autophagy-related 10.

in AGS and HGC-27 cells was 3.409 and $3.394 \mathrm{nM}$, respectively. Treatment with $3.4 \mathrm{nM}$ podofilox notably suppressed cell proliferation and colony formation compared with those observed in the vehicle control groups. Collectively, these results suggested that podofilox may be a promising drug against GC. Podofilox has been reported to disrupt the microtubules and inhibit cell cycle progression at the $\mathrm{G}_{1}$ phase (20). In addition, deoxypodophyllotoxin suppresses the cell cycle progression and promotes apoptosis in human glioma (21). Consistent with these findings, the results of the present study demonstrated that podofilox promoted cell cycle arrest at the $\mathrm{G}_{0} / \mathrm{G}_{1}$ phase in GC cells. Thus, podofilox may induce $\mathrm{G}_{0} / \mathrm{G}_{1}$ cell cycle arrest in various types of cancer cells. However, these results were insufficient to conclude whether cell cycle arrest may be a general function of the drug or if it may be cell line-specific. This should be investigated in other cancer cell lines in the future.

The molecular basis by which podofilox inhibits cancer cell proliferation and tumor growth has been investigated in previous studies. For example, podofilox inhibits HCCLM3 and HepG2 cell proliferation and migration by inactivating the PI3K/AKT/mTOR signaling pathway (22). In HCC cells, podofilox inhibits the PI3K/AKT/mTOR pathway and activates p53 (23). However, the molecular mechanisms underlying the effects of podofilox in GC remain unknown. Thus, microarray analysis was performed in the present study to determine the dysregulated genes following treatment with podofilox. A total of 1,279 genes were dysregulated in podofilox-treated AGS 
cells, including 566 upregulated and 713 downregulated genes KEGG and HALLMARK pathway enrichment analyses demonstrated that the cell cycle and the c-Myc pathway were inhibited by podofilox. By contrast, the p53 signaling pathway was activated by podofilox. Western blot analysis confirmed that the protein expression levels of c-Myc and ATG10 were downregulated in GC cells following treatment with podofilox compared with those in vehicle-treated cells. The levels of glycolysis hallmark genes HK2 and PKM2, which are frequently upregulated in cancers (24), were downregulated by podofilox compared with those in the control groups. A previously study demonstrated that ATG10 was a downstream effector of c-Myc (25). Notably, ATG10 was upregulated in GC compared with normal tissues in samples from TCGA database.

The podophyllotoxin-indirubin hybrid termed Da-1 suppresses the proliferation of leukemia cells and induces autophagy (26). The autophagy-inducing effect of podophyllotoxin has also been demonstrated in HCC cells (27). Since c-Myc signaling and its downstream effector ATG10 were both inhibited by podofilox in the present study, the involvement of ATG10 in GC cell proliferation was subsequently assessed. ATG10 was silenced in GC cells by siRNA, and the results of the CCK-8 and colony formation assays demonstrated that ATG10 knockdown significantly suppressed the proliferation of HGC-27 and AGS cells. Taken together, these results suggested that ATG10 may act as an oncogene in GC, and podofilox may suppress the proliferation of GC cells by downregulating ATG10.

In conclusion, the results of the present study demonstrated the antitumor effects of podofilox in GC. The cell cycle was arrested by podofilox at the $\mathrm{G}_{0} / \mathrm{G}_{1}$ phase, whereas apoptosis was unaffected. In addition, podofilox induced autophagy by regulating ATG10.

\section{Acknowledgements}

Not applicable.

\section{Funding}

The present study was supported by the Key R\&D and transformation program of Qinghai Province-Special project of science and technology assistance (grant no.2021-QY-213), the Applied Basic Research of Qinghai (grant no. 2018-ZJ-744), the Light of the West (grant no. 2019-33), the National Natural Science Foundation of China (grant no. 81460429), the Chunhui Plan of Ministry of Education of China (grant no. Z2017037), the Open Project of State Key Laboratory of Plateau Ecology and Agriculture, Qinghai University (grant no. 2019-ZZ-07), the Team Project of Qinghai University Medical College (grant no. 2020-KYT-2).

\section{Availability of data and materials}

The datasets used and/or analyzed during the present study are available from the corresponding author on reasonable request. The datasets generated and/or analyzed during the current study are available in the Gene Expression Omnibus repository, under the accession number GSE172408.

\section{Authors' contributions}

JA and ZS conceived the present study, performed the experiments and prepared the manuscript. YL and SD performed the cell cycle analysis. XM and LA performed the western blot analysis. Yun Y,DJ and Yup Y performed the microarray analysis. QC performed the colony formation assay. JA and ZS wrote and revised the manuscript draft. All authors read and approved the final manuscript. JA and ZS confirmed the authenticity of all the raw data.

\section{Ethics approval and consent to participate}

Not applicable.

\section{Patient consent for publication}

Not applicable.

\section{Competing interests}

The authors declare that they have no competing interests.

\section{References}

1. Joshi S and Badgwell B: Current treatment and recent progress in gastric cancer. CA Cancer J Clin 71: 264-279, 2021.

2. Ajani J, Lee J, Sano T, Janjigian Y, Fan D and Song S: Gastric adenocarcinoma. Nat Rev Dis Primers 3: 17036, 2017.

3. Wang LY, Zhao S, Lv GJ, Ma XJ and Zhang JB: Mechanisms of resveratrol in the prevention and treatment of gastrointestinal cancer. World J Clin Cases 8: 2425-2437, 2020.

4. Deng W, Jin L, Zhuo H, Vasiliou V and Zhang Y: Alcohol consumption and risk of stomach cancer: A meta-analysis. Chem Biol Interact 336: 109365, 2021.

5. Lerner B and Llor X: Genetic gastric cancer risk syndromes. Curr Treat Options Gastroenterol 18: 604-615, 2020.

6. Huang K, Ramnarayanan K, Zhu F, Srivastava S, Xu C, Tan ALK, Lee M, Tay S, Das K, Xing M, et al: Genomic and epigenomic profiling of high-risk intestinal metaplasia reveals molecular determinants of progression to gastric cancer. Cancer Cell 33: 137-150.e5, 2018

7. Machlowska J, Baj J, Sitarz M, Maciejewski R and Sitarz R: Gastric Cancer: Epidemiology, risk factors, classification, genomic characteristics and treatment strategies. Int J Mol Sci 21: 4012, 2020.

8. Shitara K, Bang Y, Iwasa S, Sugimoto N, Ryu MH, Sakai D, Chung HC, Kawakami H, Yabusaki H, Lee J, et al: Trastuzumab deruxtecan in previously treated HER2-positive gastric cancer. N Engl J Med 382: 2419-2430, 2020.

9. Liu YQ, Tian J, Qian K, Zhao XB, Morris-Natschke SL, Yang L, Nan X, Tian X and Lee KH: Recent progress on C-4-modified podophyllotoxin analogs as potent antitumor agents. Med Res Rev 35: 1-62, 2015.

10. Wang Y, Sun H, Xiao Z, Zhang G, Zhang D, Bao X, Li F, Wu S, Gao Y and Wei N: DNA damage and apoptosis induced by a potent orally podophyllotoxin derivative in breast cancer. Cell Commun Signal 16: 52, 2018.

11. Zhao W, He L, Xiang TL and Tang YJ: Discover $4 \beta-\mathrm{NH}-(6-a m$ inoindole)-4-desoxy-podophyllotoxin with nanomolar-potency antitumor activity by improving the tubulin binding affinity on the basis of a potential binding site nearby colchicine domain. Eur J Med Chem 170: 73-86, 2019.

12. Yu G, Wang LG, Han Y and He QY: clusterProfiler: An R package for comparing biological themes among gene clusters. OMICS 16: 284-287, 2012.

13. Smyth E, Nilsson M, Grabsch H, van Grieken N and Lordick F: Gastric cancer. Lancet 396: 635-648, 2020.

14. Sung H, Ferlay J, Siegel RL, Laversanne M, Soerjomataram I, Jemal A and Bray F: Global cancer statistics 2020: GLOBOCAN estimates of incidence and mortality worldwide for 36 cancers in 185 countries. CA Cancer J Clin 71: 209-249, 2021. 
15. Zhao W, Cong Y, Li H, Li S, Shen Y, Qi Q, Zhang Y, Li YZ and Tang YJ: Challenges and potential for improving the druggability of podophyllotoxin-derived drugs in cancer chemotherapy. Nat Prod Rep 38: 470-488, 2021.

16. Cohen T, Schwarz T, Vigant F, Gardner T, Hernandez R, Lee B and Tortorella D: The microtubule inhibitor podofilox inhibits an early entry step of human cytomegalovirus. Viruses 8: 295, 2016.

17. Tracz-Gaszewska Z and Dobrzyn P: Stearoyl-CoA desaturase 1 as a therapeutic target for the treatment of cancer. Cancers (Basel) 11: 948, 2019.

18. Zi CT, Gao YS, Yang L, Feng SY, Huang Y, Sun L, Jin Y, Xu FQ, Dong FW, Li Y, et al: Design, synthesis, and biological evaluation of novel biotinylated podophyllotoxin derivatives as potential antitumor agents. Front Chem 7: 434, 2019.

19. Paidakula S, Nerella S, Vadde R, Kamal A and Kankala S: Design and synthesis of $4 \beta$-Acetamidobenzofuranone-podoph yllotoxin hybrids and their anti-cancer evaluation. Bioorg Med Chem Lett 29: 2153-2156, 2019.

20. Hao SY, Feng SL, Wang XR, Wang Z, Chen SW and Hui L: Novel conjugates of podophyllotoxin and coumarin: Synthesis, cytotoxicities, cell cycle arrest, binding CT DNA and inhibition of Topo II $\beta$. Bioorg Med Chem Lett 29: 2129-2135, 2019.

21. Wang W, Gao W, Zhang L, Zhang D, Zhao Z and Bao Y: Deoxypodophyllotoxin inhibits cell viability and invasion by blocking the PI3K/Akt signaling pathway in human glioblastoma cells. Oncol Rep 41: 2453-2463, 2019.

22. Li Y, Huang T, Fu Y, Wang T, Zhao T, Guo S, Sun Y, Yang Y and Li C: Antitumor activity of a novel dual functional podophyllotoxin derivative involved PI3K/AKT/mTOR pathway. PLoS One 14: e0215886, 2019.
23. Li Y, Wang T, Sun Y, Huang T, Li C, Fu Y, Li Y and Li C: p53-mediated PI3K/AKT/mTOR pathway played a role in ptox-induced EMT inhibition in liver cancer cell lines. Oxid Med Cell Longev 2019: 2531493, 2019.

24. Guo W, Qiu Z, Wang Z, Wang Q, Tan N, Chen T, Chen Z, Huang S, Gu J, Li J, et al: MiR-199a-5p is negatively associated with malignancies and regulates glycolysis and lactate production by targeting hexokinase 2 in liver cancer. Hepatology 62: 1132-1144, 2015.

25. Sun W, Li J, Zhou L, Han J, Liu R, Zhang H, Ning T, Gao Z, Liu B, Chen X and Ba Y: The c-Myc/miR-27b-3p/ATG10 regulatory axis regulates chemoresistance in colorectal cancer. Theranostics 10: 1981-1996, 2020.

26. Wang J, Long L, Chen Y, Xu Y and Zhang L: Design, synthesis and antineoplastic activity of novel hybrids of podophyllotoxin and indirubin against human leukaemia cancer cells as multifunctional anti-MDR agents. Bioorg Med Chem Lett 28: 1817-1824, 2018.

27. Ren J, Liu Y, Li L, Zhao Y, Li Z, Wu C, Chen L and Hu K: OAMDP, a novel podophyllotoxin derivative, induces apoptosis, cell cycle arrest and autophagy in hepatoma HepG 2 cells. Cell Biol Int 42: 194-204, 2018.

This work is licensed under a Creative Commons Attribution-NonCommercial-NoDerivatives 4.0 International (CC BY-NC-ND 4.0) License. 